Musées, Patrimoine et Culture scientifiques et techniques

$126 \mid 2009$

novembre - décembre 2009

\title{
Évaluation muséale : Hermès ou les contraintes de la richesse
}

Museum Evaluation: Hermès or the confines of wealth

Joëlle Le Marec et Serge Chaumier

\section{OpenEdition \\ Journals}

Édition électronique

URL : http://journals.openedition.org/ocim/198

DOI : 10.4000/ocim.198

ISSN : 2108-646X

Éditeur

OCIM

Édition imprimée

Date de publication : 1 novembre 2009

Pagination : 7-14

ISSN : 0994-1908

Référence électronique

Joëlle Le Marec et Serge Chaumier, «Évaluation muséale : Hermès ou les contraintes de la richesse »,

La Lettre de I'OCIM [En ligne], 126 | 2009, mis en ligne le 01 novembre 2011, consulté le 01 mai 2019.

URL : http://journals.openedition.org/ocim/198; DOI : 10.4000/ocim.198

Tous droits réservés 


\title{
Évaluation muséale :
}

\section{Hermès ou les contraintes de la richesse}

\author{
Joëlle Le Marec et Serge Chaumier *
}

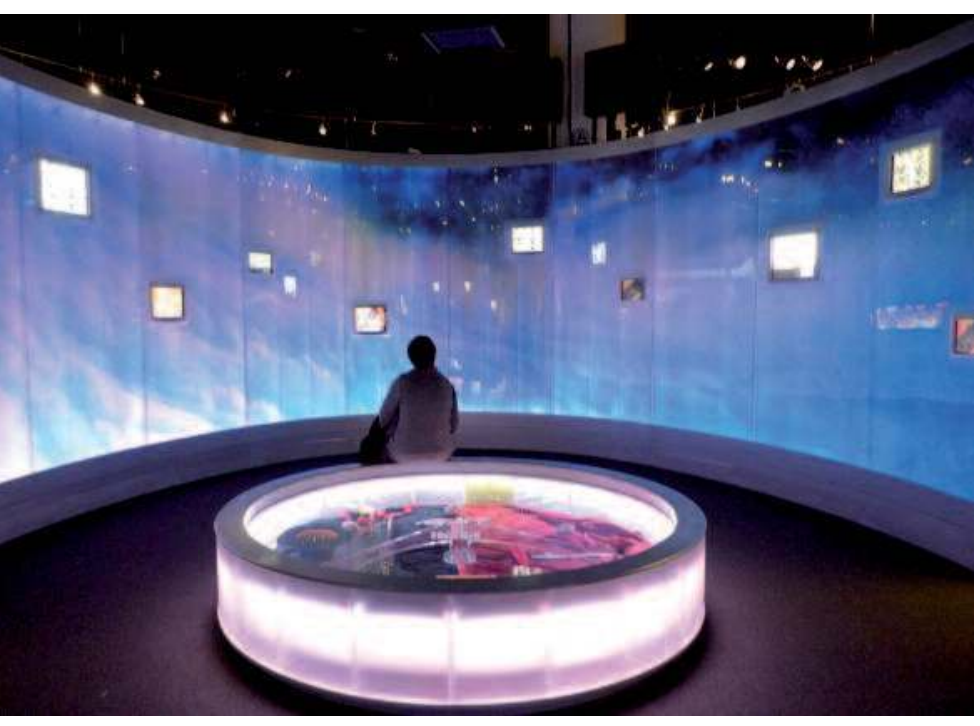

Musée national des Indiens d'Amérique à Washington (c) Serge Chaumier

* Joëlle Le Marec est professeur à l'École normale Supérieure de Lyon, directrice du laboratoire Communication, Culture et Société (JE 2419) joelle.le-marec@ens.ish.fr Serge Chaumier est directeur de l'IUP Denis Diderot

Métiers des arts de la Culture et du Patrimoine, et du Centre de Recherche sur la Culture et les Musées, équipe du CIMEOS (EA 4177), université de Bourgogne serge.chaumier@u-bourgogne.fr
Après avoir rappelé comment le terme d'évaluation s'est installé dans le monde des musées et expliqué la montée de conceptions concurrentielles de l'évaluation, les auteurs reviennent sur ses usages aujourd'hui, et présentent les démarches et les techniques de l'évaluation muséale, leurs apports mais aussi leurs dérives.

"Les évaluateurs se présentent au nom de la science, et en France, la science, c'est un signifiant-maître, on courbe la tête (...). L'évaluation n'est pas une science, mais un art du management. Sous prétexte qu'il y a de la mesure, qu'on étalonne, chiffre, compare... on simagine que c'est scientifique. Ça n'a rien de scientifique, et les meilleurs évaluateurs, les plus intelligents, qui sont aux prises avec le problème, savent parfaitement qu'il ne s'agit pas d'une science. Ce n'est pas parce qu'il y a calcul qu'il y a science ».

Jacques-Alain Miller et Jean-Claude Milner Voulez-vous être évalué? Grasset, 2006, p. 41.

Il est dans le secteur culturel un certain nombre de « mots valises »- à commencer par le mot « culture » - sorte de fourre-tout polysémique que l'on est obligé d'utiliser parce qu'ils se sont imposés à l'usage. Il vaudrait sans doute la peine d'essayer de comprendre comment ils se sont ainsi rendus incontournables, en dépit des problèmes très nombreux que pose leur manque de précision. On tente le plus souvent de conjurer les ambiguïtés en comptant sur les contextes 
d'usage et les compréhensions tacites dans une même communauté. Les problèmes deviennent plus aigus quand ces communautés se reconfigurent, quand les domaines concernés évoluent, quand leurs frontières éclatent.

La notion d'« évaluation » fait partie de ces termes et nombreux sont les commentateurs qui en ont regretté l'usage jusque-là indépassable. Ce qui était déjà problématique du fait de l'évolution historique du concept dans le secteur muséal le devient davantage encore à l'heure où le mot dépasse très largement la seule attribution qui en était donnée jusque-là. Le terme «évaluation » dans le monde muséal correspond à plusieurs types de démarches, dont certaines ont été construites dans la durée et ont évolué peu à peu, et d'autres sont importées récemment de secteurs qui ont très peu à voir avec les logiques muséales.

Lévaluation des connaissances, importée du monde scolaire s'est installée très tôt au musée mais elle s'y est transformée profondément ${ }^{(1)}$. Le mot s'est chargé de nouvelles significations, plus ouvertes mais aussi plus floues que celles qu'il avait en didactique. L'« évaluation d'expositions » a fait exploser le spectre des usages du terme : en effet, l'exposition déborde à tel point la question de transmission de connaissances, que les démarches d'évaluation didactique ont été modifiées par la richesse de ce que les visiteurs ont pu répondre lors des enquêtes. On a ainsi découvert de multiples dimensions de l'expérience de visite ${ }^{(2)}$.

Lévaluation a contribué à unifier une conception générale de l'exposition comme dispositif de communication, et en particulier comme discours (3). Le concept a trouvé alors un emploi large et généreux qui dépassait très largement ce qu'il sous-entendait $a$ priori. Plus récemment, d'autres influences sont intervenues : la rencontre entre l'évaluation muséale et l'évaluation des politiques publiques d'une part, avec l'évaluation importée du domaine du marketing d'autre part. Depuis quelques années, c'est l'évaluation gestionnaire, inspirée des techniques de management, qui tend à entrer au musée. Peu importait il y a vingt ans que l'évaluation soit un terme utilisé avec des sens très différents au musée et dans l'entreprise, puisqu'il y avait peu de chance que des professionnels des deux secteurs aient à se comprendre à propos de ce terme en particulier. Mais aujourd'hui, un même terme recouvre des démarches qui servent des objectifs totalement différents, parfois contradictoires.

Nous voudrions dans ce texte, non pas présenter ces différentes démarches comme s'il s'agissait d'une gamme d'outils équivalents, également intéressants, mais revenir sur la spécificité et la richesse de l'évaluation telle qu'elle s'est développée dans le milieu muséal et les centres des sciences, et ainsi, aider à éviter les confusions qui peuvent naître des usages du terme dans le contexte actuel qui est celui d'une transformation des communautés professionnelles impliquées dans la vie des musées, et des frontières du «monde du musée ».

\section{Quelques rappels historiques: le souci du public et le développement de l'évaluation muséale}

Née au début du siècle dernier dans les musées de science américains, à partir de travaux pionniers, l'évaluation muséale connaît une expansion continue depuis trente ans, essentiellement depuis la fin des années 1980 en France (4). Il n'est pas anodin de remarquer que ce développement coïncide avec ce qu'on a appelé « le boom des musées » sous l'impulsion conjointe d'une politique culturelle et d'une politique scientifique très volontariste. Le ministère de la Culture s'implique fortement dans une politique de rénovations et de créations (chantier du Grand Louvre, rénovation des musées de province). Le ministère de la Recherche quant à lui, intègre l'obligation de vulgariser dans le statut des enseignantschercheurs et s'investit très massivement, après le colloque "Chevènement " (1982) dans le soutien au développement en cours des centres de culture scientifique et technique, dont la Cité des Sciences et de l'Industrie.

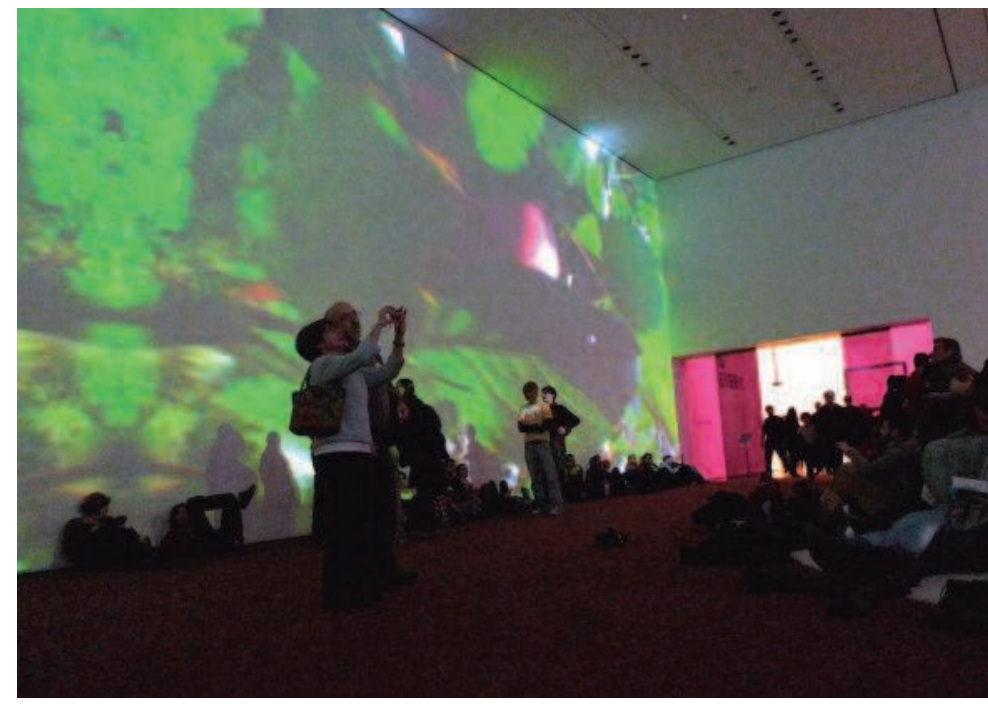

Musée d'Art moderne de New York ๑) Serge Chaumier 
L'évaluation provient essentiellement du besoin de comprendre et prendre en compte des publics, dans la perspective de développer le rôle social du musée. Cette préoccupation naît d'un nouveau regard sur les musées qui se forge à partir des années 1960 et aboutit dans les années 1980 au mouvement de la Nouvelle Muséologie (5).

La critique de l'institution accompagne la réflexion concernant les outils nécessaires à l'appropriation de la culture, sur lesquels se penchent les sociologues, de Joffre Dumazedier à Pierre Bourdieu (6). Cette critique académique accompagne une dynamique nouvelle chez les professionnels des musées, en faveur d'une muséologie proche des enjeux sociaux. Cette dynamique est internationale. Elle se traduit en France par la création de l'association " Muséologie nouvelle et expérimentation sociale » et le mouvement des écomusées. Elle catalyse le développement du secteur de la médiation culturelle, non seulement dans les musées de science, mais dans l'ensemble des musées. Celle-ci inspire la conception des expositions thématiques scénographiées et la programmation d'animations associées, notamment dans le secteur des sciences et techniques, et toutes les formes de propositions d'aide à la visite, à l'interprétation des œuvres et à l'appropriation des musées ${ }^{(7)}$.

La préoccupation du public se traduit donc par une combinaison d'intérêts et d'initiatives académiques et professionnelles, assez proche de ce qui s'est passé au moment du développement de la lecture publique. Le secteur des études et recherches se développe, et combine des intérêts de connaissances académiques et muséologiques. L'association Expomédia (Peuple et Culture) réunit des professionnels des musées, des artistes, des architectes, et les chercheurs qui créeront les formations interdisciplinaires de muséologie à l'université. D'abstrait et homogène, le public s'impose comme une réalité vivante, foisonnante, au travers de recherches universitaires et d'études réalisées par les musées. Ces travaux en décrivent la diversité, le suivent dans la durée, en comparent la composition et les pratiques d'un lieu à l'autre.

Des colloques et journées d'études réunissent des scientifiques, des conservateurs, des médiateurs et des muséographes à partir des années $1980{ }^{(8)}$. Ces moments de rencontre permettent de multiplier les échanges d'expériences et la confrontation des connaissances sur le public. Celui-ci prend corps non seulement dans les caractéristiques très partielles et spécifiques par lesquelles l'approchent le médiateur, le directeur de musée, le sociologue, le concepteur multimédia, mais par l'ensemble de ces points de vue sans cesse discutés et échangés ${ }^{(9)}$.

\section{La montée du marketing et la culture managériale}

Pendant cette période de développement intense qui implique essentiellement les "gens de musées », les universitaires, les médiateurs et les visiteurs, deux autres dynamiques externes au musée vont converger vers l'institution en y important des intérêts et des conceptions très différents, même s'ils vont renforcer dans un premier temps l'apparence d'un consensus général pour développer les études de public.

Une conception managériale des établissements du service public (notamment les secteurs de la santé et de la culture) va se développer dans les milieux de l'administration publique, avec l'idée de contrôler et d'évaluer les résultats des efforts financiers de l'État et des collectivités. Les normes de gestion vont s'imposer peu à peu et culminer avec la Loi Organique relatives aux Lois de Finance (LOLF) en 2001.

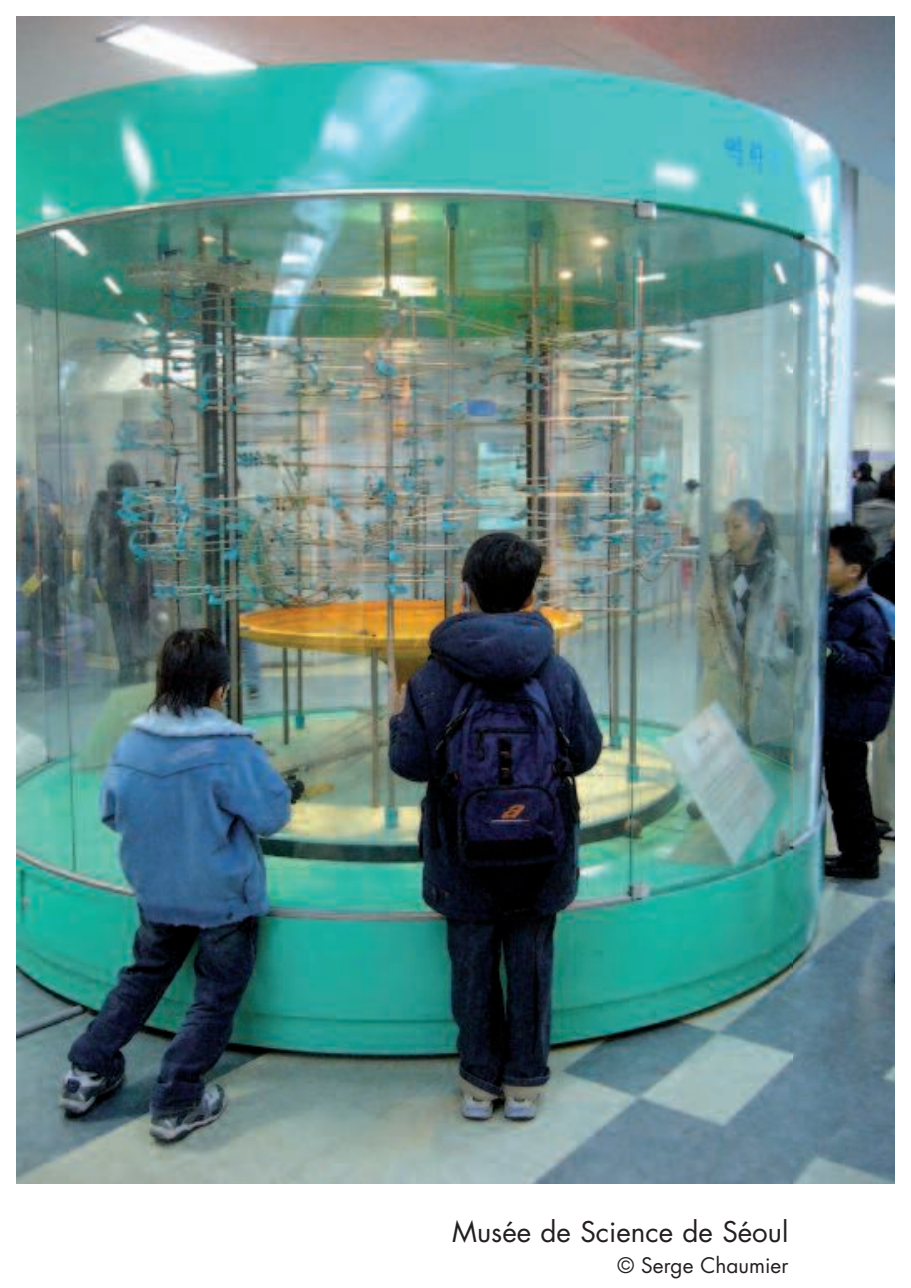


L'effort porte sur la création d'indicateurs de la performance. Le public va être considéré comme un des indicateurs privilégiés : un musée ou un hôpital sont des entreprises, qui ont une production, caractérisée par l'existence d'une « consommation » par les clients ou usagers. Dans cette vision la fréquentation devient un des indicateurs de performance des musées, dans une toute autre perspective que celle qui inspire l'évaluation muséale. La grille appliquée sur le phénomène de la visite est réductrice et rigide, mais la force appliquée pour modeler l'objet auquel elle s'applique - le musée - est considérable.

Le marketing est également une dynamique a priori exogène, mais dont la greffe sur le musée, moins contrainte que les normes de management, a été catalysée par la dynamique muséale des années 1980 que nous avons évoquée (10). Les musées, préoccupés par l'innovation muséographique et médiatique ont fait appel largement au secteur de la communication professionnalisée. Graphistes, scénaristes, professionnels des médias, de l'audiovisuel et de la publicité, s'impliquent profondément dans l'ensemble des strates du travail muséal au quotidien. Ils contribuent à diffuser des modèles et un lexique familier au champ de la communication et des médias, avec en tête, en permanence, l'enjeu d'une communication réussie avec le public considéré comme ensemble de récepteurs : il s'agit de diffuser des messages, d'atteindre la cible, de créer une image... Ce modèle se prête bien à l'idée du travail muséal comme production médiatique. Il converge aisément à la fois avec la conception managériale qui est développée dans les strates de la direction et du pilotage politique des musées, et dans les strates de la médiation culturelle.

Ce rappel évidemment trop succinct de l'évolution récente du secteur est nécessaire pour comprendre la montée de conceptions concurrentielles de l'évaluation. Il nous faut maintenant développer plus en détail en quoi consistent techniquement les protocoles particuliers d'évaluation muséale.

\section{L'évaluation muséale : la découverte de l'exposition comme dispositif pluriel}

Au-delà de la simple connaissance quantitative des compositions et répartitions des différentes catégories de public, selon les caractéristiques sociodémographiques classiques, en termes d'âge, de sexe, d'origine géographique, de niveau d'étude ou de professions, des modalités de visite, des caractéristiques des visiteurs, ou encore de leurs habitus vont donner lieu à des recensements statistiques éventuellement adaptés à la spécificité de chaque musée. Cette connaissance que l'on peut considérer comme basique s'avère indispensable pour conduire des politiques d'établissement ciblées et efficaces (politique de l'accueil, politique tarifaire, aménagements des lieux, communication, actions culturelles...).

Mais si la connaissance quantitative est incontournable, elle demeure grossière pour saisir certains aspects plus subjectifs, telle que les motivations, les appréciations, les intentions, les représentations, et même la finesse de certains usages et actions du visiteur dans l'espace d'exposition. Pour cela, les techniques d'évaluation vont se déployer pour affiner les connaissances acquises avec des méthodes dites qualitatives, recourant par exemple aux entretiens, aux observations, à l'analyse sémiologique d'un corpus donné. Si l'évaluation tire son nom du souci premier de mieux saisir les acquisitions par les visiteurs par des tests de connaissances pratiqués au sortir d'une exposition, très vite elle va dépasser cet aspect trop restrictif, à l'intérieur même du cadre didactique. Comment tenter d'objectiver un apprentissage dans l'exposition ? La mémorisation ou la reformulation sont-elles pertinentes dans ce contexte pédagogique informel ? L'impression de découverte, la nature et la qualité des questions ne sont-elles par des indicateurs plus adaptés ? Quel est le point de vue des visiteurs eux-mêmes sur leur propre activité au musée et notamment, sur ce qu'ils ont appris (11) ?

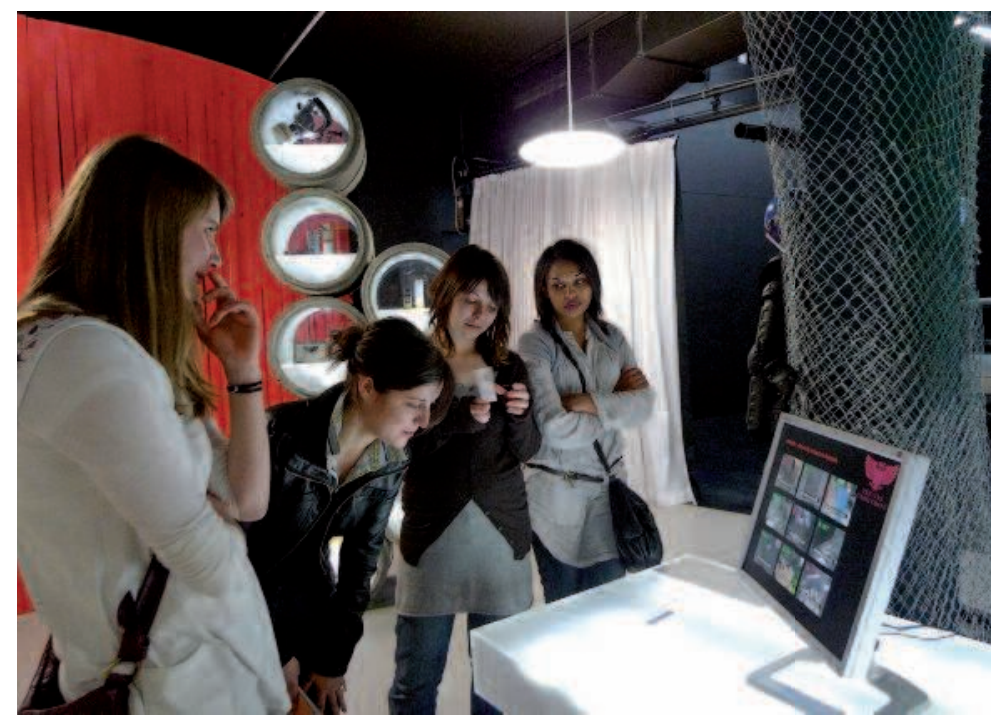

Exposition La Marque jeune au musée d'Ethnographie de Neuchâtel ○ Serge Chaumier 
Les musées de science vont légitimement être les premiers à développer le spectre des démarches. Certains ne peuvent se souvenir en effet que de cette mission pédagogique, les enjeux de conservation étant bien moindres que dans les musées d'art. Mais ce qui était, et demeure encore souvent une demande initiale de la part des concepteurs (« qu'est-ce que les visiteurs ont appris ou retenu de mon exposition?»), ouvre sur des questions plus générales, qui intègrent le point de vue des visiteurs et pas uniquement les objectifs des concepteurs. La façon dont les visiteurs se sont appropriés les lieux ne correspond que rarement à la projection que l'on avait initialement envers un « visiteur idéal », son parcours, ses actions, mais aussi les interactions avec les expôts et avec les autres membres du groupe de visite, voire avec les autres visiteurs. Plus encore, la façon dont le visiteur s'approprie les contenus dépasse de loin la seule vérification des connaissances retenues. L'évaluation va montrer que si le concepteur transmet, il a aussi beaucoup à apprendre du visiteur qui interprète les propositions culturelles en y apportant beaucoup de sa propre histoire, de sa culture, de ses aspirations. La démultiplication des usages constatés et des réinvestissements de sens à l'intérieur des espaces d'exposition, mais aussi au-delà après la visite, ouvre sur une vision plurielle de l'exposition.

Les enquêtes menées dans le cadre de l'évaluation muséale vont souvent faire changer la pratique même de conception de l'exposition. Les concepteurs se sentent parfois inspirés par la figure de cet interlocuteur passionné qui émerge des études. Loin de développer la peur du client insatisfait ou indifférent, ou la crainte d'un alignement démagogique des contenus sur des attentes, les études peuvent parfois faire apparaitre un rapport de confiance particulièrement émouvant et rassurant pour les concepteurs (12).

Les études peuvent favoriser une vision informée du spectre de ce qui est possible, avec des expositions plus polysémiques, voire co-construites, car achevées seulement par l'usage que le visiteur en réalise. Le cas extrême est représenté par l'exposition qui s'élabore comme un work in progress, s'inventant au travers de l'action même des visiteurs. Plus simplement on peut voir dans la façon de construire les parcours et dans les propositions spécifiques (textes, manipulations interactives, audiovisuels), une résultante des connaissances acquises par les études qui dévoilent des logiques d'usages et de réappropriations par les visiteurs. Le cas du texte d'exposition est emblématique de ce que l'évaluation d'exposition a pu apporter pour améliorer les pratiques, car il y a tout un registre de savoirs accumulés par les études conduites, pour écrire des textes d'exposition opérationnels. La façon d'écrire, le vocabulaire utilisé, les formulations, l'ampleur du texte, la composition graphique, les tailles de caractères comme les couleurs retenues, l'emplacement et la progression, sont autant de données sur lesquelles l'évaluation a apporté des résultats probants (13). Il est à ce titre étonnant de constater encore que ces acquis ne soient pas mobilisés par certains concepteurs. La même constatation peut du reste être faite pour ce qui concerne les audiovisuels, le multimédia, ou encore la disposition des éléments expographiques.

\section{Modes de faire et méthodes}

À chaque problématique posée, ses méthodes d'enquête adéquates. Il n'y a pas une, mais de multiples façons de conduire une évaluation selon les questions que l'on se pose, et donc les résultats auxquels on veut aboutir. Il n'est pas le lieu pour les détailler ici, mais il convient de signaler seulement que c'est souvent une combinaison de techniques qui est utilisée, et que le

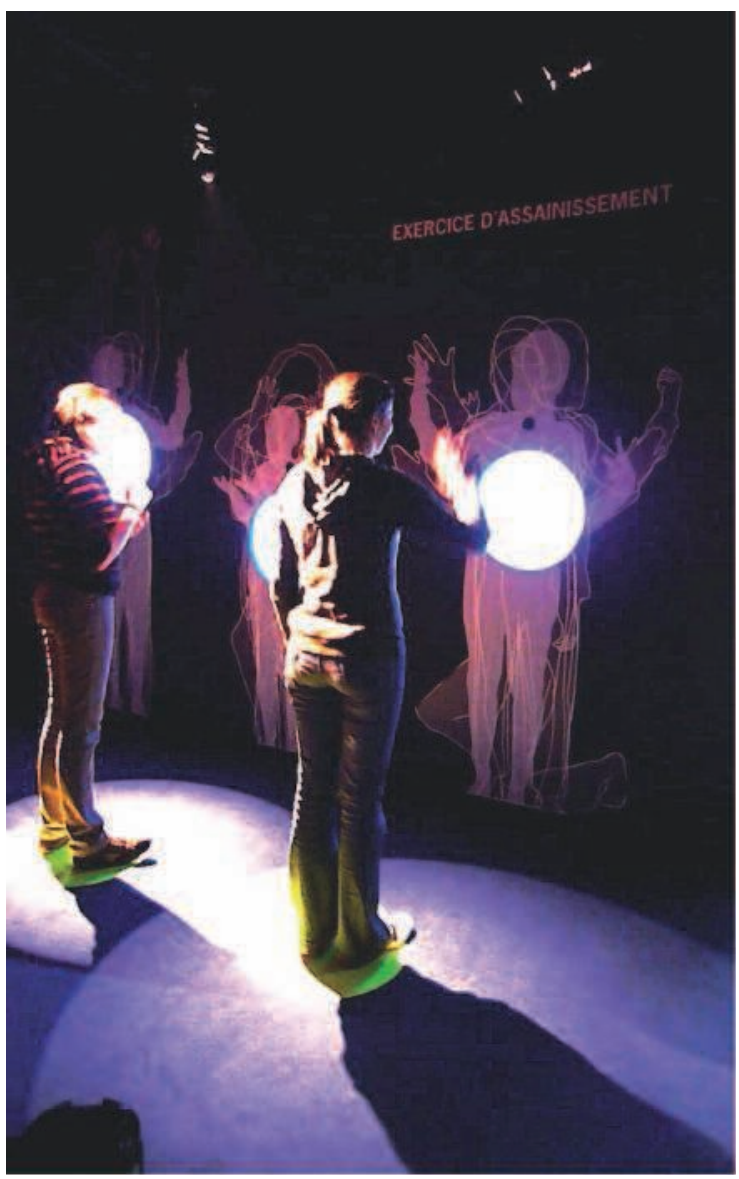

Exposition $\mathrm{H} 2 \mathrm{O}$ au Compa à Chartres (C) Serge Chaumier 
«bricolage méthodologique », au sens de Levi-Strauss est d'une grande richesse heuristique. L'observation, qu'elle soit active ou passive, combinée à l'entretien, quil soit individuel ou collectif, offre des ressources inépuisables. Nombre d'évaluations ont ainsi expérimenté des formes nouvelles, recourant à la photographie ${ }^{(14)}$, à la vidéo, aux entretiens en cours de visite, post-visite, aux tests, aux entretiens de groupes, aux comités de visiteurs, à la mise en situation à partir de prototypes, aux recherches actions... Sans entrer dans le détail, signalons seulement que la méthode doit être pensée à chaque fois pour être adaptée au mieux aux questions posées initialement en fonction du contexte particulier, dû à l'histoire du site, à sa topographie, aux propositions culturelles déployées, à ses ressources humaines, financières et logistiques. Il peut donc sembler difficile de tirer de la diversité des études des généralités concernant les pratiques des visiteurs. Pourtant, au fil des études, des constantes et des convergences apparaissent : l'effort souvent sous-estimé des visiteurs pour tenter de comprendre ce qu'on a voulu leur dire, l'importance des pratiques de sociabilité, les logiques de découverte et de reconnaissance.

Il est admis de décrire en trois grandes catégories les évaluations conduites dans les expositions :

- sommatives pour décrire les études conduites une fois l'exposition ouverte au public,

- formatives pour désigner celles conduites au moment de la conception même de l'exposition,

- préalables pour regrouper celles que l'on mène avant même la conception, en amont du scénario, afin d'apporter des éléments de réflexion qualitatifs à l'équipe de conception.

Si les évaluations de texte sont exemplaires de ce que peut apporter l'évaluation formative pour aider à la conception, les études préalables permettent de développer une « culture du visiteur » à partir d'une compréhension fine de ce que les visiteurs associent à certains thèmes, mais surtout, de ce qu'ils pensent être la mission du musée lorsque celui-ci traite certains thèmes sensibles : le musée se voit opposer les médias, on attend de lui qu'il puisse être une référence fiable, dans un environnement social saturé de discours qui inspirent une certaine méfiance.

Les résultats des études préalables peuvent aussi susciter un véritable dialogue en différé, les concepteurs répondant à certaines questions apparues lors des enquêtes, voire citant, même implicitement, des éléments exprimés par ceux-ci. Mais surtout, ces évaluations permettent de combattre en permanence les idées reçues souvent étonnamment fausses à propos du public, de ses attentes, de ses pratiques, de ses réactions. Si les résultats de l'évaluation servent indéniablement à améliorer les conditions de visite, et donc le confort des visiteurs, et ainsi à rendre plus efficace le fonctionnement de l'établissement, l'évaluation ne saurait être limitée à cela.

Les actions dites de « remédiations » qui consistent à améliorer les dispositifs suite aux résultats de l'évaluation, sont nécessaires et ont des retombées intéressantes. Éviter par exemple que les gens se perdent et leur épargner une fatigue inutile est loin d'être négligeable. Cependant, du point de vue même des visiteurs, la visite ne se réduit pas à un bon moment passé le plus confortablement possible. Au contraire : la tolérance à l'inconfort est beaucoup plus grande que la tolérance au mélange des genres. Les visiteurs sont prompts à repérer les signes d'une "fausseté » dans l'accueil ou la démarche de conception.

L'évaluateur apporte des connaissances plus fondamentales aussi sur le rapport entretenu par les visiteurs au site, au sujet présenté et aux actions conduites. Il est ce tiers qui met en dialogue des acteurs qui ne sont pas nécessairement appelés à se rencontrer, concepteur, conservateur, publics fidèles et publics occasionnels, voire non-publics, éloignés de l'institution. En faisant circuler la parole entre les acteurs, en faisant remonter au concepteur des logiques qui ne sont pas les siennes, il éclaire le rapport complexe et ténu, pluriel, qui s'établit nécessairement entre des publics et des propositions culturelles.

\section{Des apports remis en cause}

Si l'évaluation muséale est en phase avec les impératifs professionnels d'amélioration des savoir-faire de muséologie et médiation, il ne s'agit pas pour autant de coller strictement à des attentes. En effet, les publics apprécient justement le fait de ne pas être engagés dans un rapport de type « offre/demande » avec le musée. Plus fondamentalement, il ne s'agit pas pour les visiteurs d'user de produits ou services, mais de se prêter à ce qui a été prévu pour eux par le musée. Les visiteurs s'attendent à certaines choses plus qu'ils ne les attendent. Le principe de la pratique est en effet une confiance dans ce que le musée propose. Encore une fois, de ce point de vue, l'évaluation est moins un outil pour améliorer une production qu'un moyen d'anticiper et d'entretenir une communication permanente avec des publics.

Il existe cependant un facteur de changement important : la pratique de visite, le sens de celle-ci, le lien à l'institution, ne sont pas de même nature 


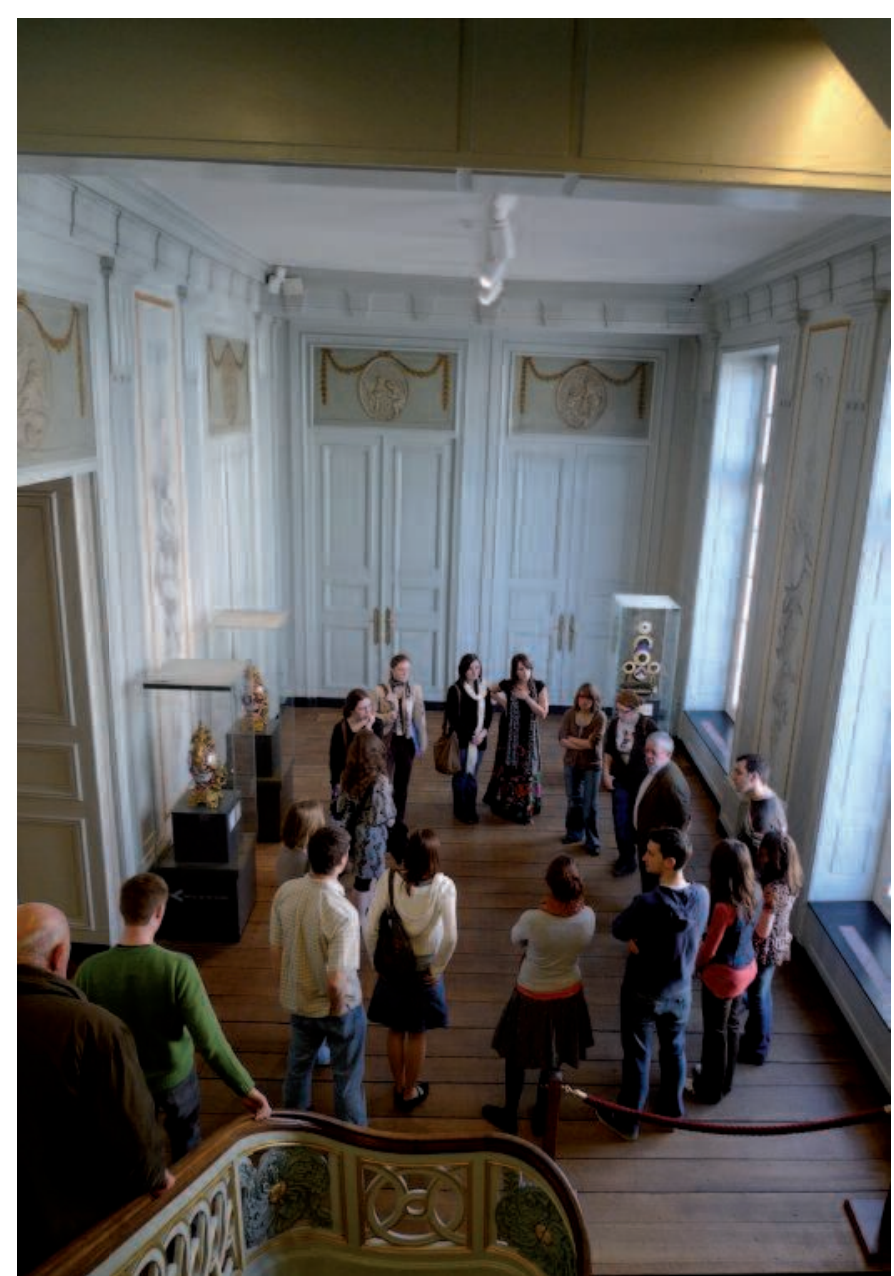

Musée d'Histoire « Le Grand Cursius » à Liège (c) Serge Chaumier

selon que l'on est touriste, touriste étranger qui plus est, ou membre de la communauté politique et culturelle à laquelle le musée appartient. Le touriste étranger peut fort bien ne pas se sentir engagé dans la relation à une institution culturelle, mais se percevoir comme l'usager d'une offre disponible, pour son agrément. C'est donc cette fraction du public qui peut amener à justifier le rabattement de l'évaluation à une technique d'adaptation d'un service à une clientèle. La conception marketing a ainsi tendance à gagner du terrain par les grands musées accueillant de très nombreux touristes, et cela d'autant plus que cette dynamique va dans le sens du développement d'une économie de la culture et du tourisme, et d'une mise en cause de l'autonomie des missions institutionnelles par rapport aux appétits des acteurs du marché. Une conception qui a tendance à gagner du terrain d'autant plus facilement que la confusion envers le sens de l'action culturelle est grande.
Même si des décennies d'études et recherches ont montré qu'il est absurde de parler de « clients », de «produits » et de « consommation» dans un secteur pour lequel ces concepts n'ont pas de pertinence (pas plus qu'ils ne le sont pour appréhender la religion et les rituels), le fait est qu'une partie des professionnels de la culture sont parfois amenés à considérer cette évolution comme inéluctable tant les pressions exercées sont fortes. Dès lors, l'évaluation muséale peut se voir ramenée à une technique permettant de rationaliser la production d'une offre. La notion d'évaluation se referme sur des acceptions restrictives, qui ne reprennent pratiquement rien des questions ou des résultats issus de l'évaluation muséale, ni même de l'évaluation didactique.

Hermès prend alors sa signification plus récente, de dieu du commerce, laissant oublier son origine lointaine de dieu passeur des âmes des morts, communicateur des passions humaines et divines, médiateur entre le monde céleste et terrestre, accompagnateur des voyageurs dépassant les frontières. Bref, une allégorie semble t-il d'une évolution actuelle du monde de la culture.

Nouveau credo des politiques, l'évaluation de l'efficacité des institutions au nom du contrôle des finances, de la bonne gestion et dépense de l'argent public, vient désigner le secteur culturel comme un champ d'activité comme un autre. Longtemps l'exception culturelle a porté sur le fait que la dépense culturelle bénéficiait d'une spécificité qui en interdisait précisément la pure mesure comptable, comme le mentionnaient les ministres, de Malraux à Jacques Duhamel. Ce temps semble révolu, puisque des critères le plus souvent d'ordre quantitatif sont désormais appliqués dans une logique de contrôle. Cette évolution s'inscrit du reste dans une tendance plus générale, qui touche l'ensemble du secteur public.

Il est donc essentiel pour les institutions muséales de prendre la mesure de ces différentes conceptions de l'évaluation pour se garder des risques de dérives que le terme peut charrier dès lors qu'il est manié ou utilisé pour des finalités différentes. Il importe de rappeler pour différencier ces catégories que l'évaluation muséale résulte toujours d'une démarche volontaire dont l'objectif est une collaboration entre un commanditaire et un évaluateur afin de progresser ensemble dans la connaissance des publics.

Les évaluations peuvent servir différentes finalités et l'on voit bien que ces méthodes sont utilisées pour produire des données, qui peuvent ressortir du simple registre des connaissances ou fournir des indicateurs 
de contrôle. Les sciences sociales sont particulièrement en prise, depuis leur origine, avec ces deux types d'usages. Les informations collectées et produites peuvent être inventoriées, classées et documentées pour alimenter une banque de données, un lieu de ressources, appelé aussi parfois " observatoire ». Comme l'évaluation, il en est de plusieurs espèces, qui servent d'outil de contrôle, de support d'information ou de lieu de médiatisation et de transmission entre des acteurs. De la synergie entre des partenaires rassemblés autour d'une même préoccupation peut naître un dynamisme que seul le collectif permet d'entrevoir. Un observatoire est un formidable outil pour faire progresser les connaissances en mettant en perspective et en offrant un centre de ressources et de comparaison des données. À partir de là, de nouvelles approches peuvent s'opérer et un approfondissement s'accomplir. En alimentant un observatoire de la culture scientifique, où les travaux issus de l'évaluation muséale trouvent place, parions que l'OCIM puisse à l'avenir proposer un lieu de stimulation pour l'esprit et une occasion de valorisation et de création de nouvelles connaissances pour le monde des musées.

\section{Notes}

(1) Voir notamment Guichard, J. et Martinand, J.-L. Médiatique des sciences. Paris : PUF, 2000.

(2) Dès 1987, Hana Gottesdiener dresse un inventaire des types d'évaluation au musée. Voir Gottesdiener, H. Évaluer l'exposition. Paris : La Documentation française, 1987.

(3) Le numéro 2 de la revue Publics \& Musées, paru en 1992, contient deux articles, de Jean Davallon et de Bernard Schiele qui témoignent parfaitement de ce lien entre évaluation et conception de l'exposition comme média.
(4) Voir Chaumier, S. Les méthodes de l'évolution muséale, la Lettre de l'OCIM, n65, 1999.

(5) Voir les textes choisis et présentés par André Desvallées dans Vague : une anthologie de la nouvelle muséologie. Mâcon : W/MNES, 1992.

(6) Voir Dumazedier, J. Vers une civilisation du loisir ? Paris : Seuil, 1962, réed 1972 ; Bourdieu, P., Darbel, A. et Schnapper, D. L'Amour de l'art. Les musées et leur public. Paris : Minuit, 1966, 1969.

(7) Il n'est pas possible de rendre compte de la richesse considérable des travaux inspirés par la médiation, surtout prise au sens large, au-delà d'une réduction au secteur de la création d'un métier de médiateurs face au public. Voir notamment la revue $M E I, \mathrm{n}^{\circ} 19$, « Médiations et médiateurs ». L'Harmattan, février 2004.

(8) En 1992, par exemple, la Cité des Sciences organise des journées d'études sur l'évaluation muséale. Au même moment, le programme REMUS, de soutien à la recherche en muséologie, présente les travaux réalisés par les équipes de chercheurs et de professionnels.

(9) La Lettre de l'OCIM est également un lieu d'expression et de partage important sur ces questions.

(10) Voir le numéro 11-12 de la revue Publics E Musées, « Musées et marketing », 1997.

(11) Voir par exemple Guichard, J. Nécessité d'une recherche éducative dans les expositions à caractère scientifique et technique, Publics $\&$ Musées, n7, 1995, pp. 95-115.

(12) Voir Le Marec, J. Publics et musées, la confiance éprouvée. Paris : L'Harmattan, 2007.

(13) Voir notamment les travaux de Daniel Jacobi et Marie-Sylvie Poli ; Poli, M.-S. Le texte au musée : une approche sémiotique. Paris : L'Harmattan, 2002.

(14) Voir Davallon, J. et Gottesdiener, H. Le musée national des techniques sous l'œil de ses visiteurs, La Revue du musée des Arts et Métiers, $\mathrm{n}^{\circ} 1$, septembre 1992 . 